\title{
Interactions between an endogenous oscillator and response to tap in $C$. elegans
}

\author{
SUSAN SIKLOS, JENNIFER A. JASPER, STEPHEN R. WICKS, and CATHARINE H. RANKIN \\ University of British Columbia, Vancouver, British Columbia, Canada
}

\begin{abstract}
In this study, we investigated the interaction between the endogenous oscillator governing defecation in Caenorhabditis elegans and the worm's response to a mechanical stimulus, a tap. The results showed that the defecation cycle became progressively longer over the life span of the worm. Taps changed the phase of the defecation cycle, and taps delivered at different phases of the defecation cycle had significantly different effects on the phase change observed. In contrast, the phase of the defecation cycle in which the tap was delivered had very little effect on either the frequency or the magnitude of the response to the tap. The phase change by the taps habituated slowly over multiple taps. There was no effect of tap delivery at a consistent phase of the defecation cycle; therefore, interaction between tap and defecation cannot account for much of the variance seen in a normal habituation experiment.
\end{abstract}

One of the simplest but least understood forms of learning is habituation, which is defined as a decrease in responding over repeated stimulation (Groves \& Thompson, 1970). In many organisms, a striking feature of habituation is that there are large individual differences; thus, the mean curves are not smooth but show a high level of variability. For examples, see the data from Aplysia (e.g., Rankin \& Carew, 1987), from Caenorhabditis elegans (C. elegans; e.g., Rankin \& Broster, 1992), from fish (e.g., Peeke \& Veno, 1973), and from rats (e.g., Davis, Parisi, Gendelman, Tischler, \& Kehne, 1982). One possible hypothesis for the within-subjects variability observed over trials and the "noise" seen in the group mean habituation curves in response habituation is that they reflect the effects of the stimulus' interacting with different phases of an endogenous oscillator.

C. elegans offers a good model system in which to study organismal factors affecting habituation, because its physiology, genetics, and developmental stages are well known. C. elegans has a small nervous system consisting of only 302 neurons and, therefore, is a prime candidate for determining which cells are involved in learning. One stimulus, which has been used in studies of habituation in C. elegans, is the tap stimulus (Rankin, Beck, \& Chiba, 1990; Rankin \& Broster, 1992; Wicks \& Rankin, 1995). In response to a vibratory stimulus (a tap) to the Petri dish, adult $C$. elegans swim backward. This response, which has been named the tap-withdrawal response (Chiba \& Rankin, 1990), shows habituation to repeated stimulation. Although different groups of subjects trained with different interstimulus intervals (ISIs; the

This work was funded by an NSERC operating grant to C.H.R. and an NSERC Predoctoral Fellowship to S.R.W. Correspondence concerning this article should be addressed to C. H. Rankin, Department of Psychology, University of British Columbia, Vancouver, BC V6T 1Z4. Canada (e-mail: crankin@cortex.psych.ubc.ca), times between stimulations) show systematic and significant differences in their level of habituation performance, individual subjects within any given group show a high degree of variability across trials. This individual variability cannot be accounted for by innate or experiential differences among subjects, because the subjects are (1) genetically identical, (2) raised in similar conditions, (3) evaluated according to a strict and precise protocol at the same developmental stage, and (4) tested with the same equipment (Galloway, Wicks, \& Rankin, 1997).

One hypothesis to explain the within-subjects variability observed over trials in habituation (not only in C. elegans, but in many organisms studied) is that it reflects the effects of an interaction of response to tap with an endogenous oscillator. It is possible that the magnitude of a given response might vary if the stimulus is delivered at different phases of some endogenous oscillator. At some phases of the oscillator, responses may be larger. at others smaller, than the average response. It is possible that some reported cases of sensitization might simply be the result of a chance interaction between the time intervals of the experimental design and the peak of an internal oscillator. In studies of habituation in which stimuli are delivered at a set interval, it may be that this interval conflicts/interacts with an internal oscillator in such a way that variations in response amplitude are greater than they would be if all the stimuli occurred at the same phase of the oscillator.

C. elegans has several behaviors that are periodic (i.e., feeding, defecation, and egg laying) and possibly controlled by endogenous oscillators (Liu \& Thomas, 1994). The variability observed in studies of habituation to tap may be the result of an interaction between the animal's ability to respond to tap and differing states of an internal oscillator. The interaction between the tap response and the endogenous defecation cycle was chosen for these experiments because the defecation cycle of wild-type 
worms is highly periodic, with deviations of only a few seconds (Croll \& Smith, 1978; Liu \& Thomas, 1994; Thomas, 1990), and is within the same time frame as the $10-60 \mathrm{sec}$ ISIs used in many habituation studies (e.g., Rankin et al., 1990; Rankin \& Broster, 1992; Wicks \& Rankin, 1995). The defecation cycle of $C$. elegans lasts, on average, 45-70 sec and ends with the expulsion of waste from the body (defecation). Defecation itself consists of three steps: the contraction of the posterior body muscle, followed by the contraction of the anterior body muscle, and ending with the contractions of the intestinal muscles and the anal depressor, which results in the expulsion (Thomas, 1990). Together, these muscle contractions signal that a defecation has occurred; thus, the defecation cycle of $C$. elegans is easy to identify and can be accurately scored. In addition, the behavior and genetics of the defecation cycle are well understood (Iwasaki \& Thomas, 1997; Liu \& Thomas, 1994; Reiner \& Thomas, 1995; Reiner, Weinshenker, \& Thomas, 1995; Thomas, 1994). Finally, a touch stimulus, which shares much of the same neural pathway as the tap stimulus (Wicks \& Rankin, 1995), resets the defecation cycle (Liu \& Thomas, 1994; Thomas, 1990). It is not known whether a different mechanosensory stimulus, the tap stimulus, also produces a reset in the defecation cycle. The present experiments were designed to investigate the effects that tap has on the defecation cycle in C. elegans, the effect the defecation cycle has on the response to tap, and the interaction between the defecation cycle and the processes of habituation.

\section{GENERAL METHOD}

\section{Subjects}

A total of 458 C. elegans hermaphrodites from the Bristol N2 wild-type laboratory strain (Brenner, 1974) were used. The subjects were obtained from the Caenorhabditis Genetics Center and were grown synchronously at $20^{\circ} \mathrm{C}$ on $5-\mathrm{cm}$ Petri plates that were filled with 10-ml Nematode Growth Medium (NGM) agar and streaked with E. coli strain OP50 (Brenner, 1974).

\footnotetext{
Apparatus

The animals were observed on the NGM (Brenner, 1974) agar with a stereomicroscope (Wild Leitz, Canada, Ltd., Model M3Z). For data collection and scoring purposes, video tape-recording equipment (Panasonic camera D5000, Panasonic 1960 VCR, NEC color monitor) was attached to the stereomicroscope. In order to time the stimulus delivery and the defecation cycles, a time-date generator (Panasonic 810) superimposed a stopwatch onto the monitor. During testing, the Petri plates that contained individual worms on NGM agar were held by a holder designed out of a Petri plate lid that had been mounted onto a plastic rod. The rod was attached to a Marzhauser micromanipulator (Model MM33) for smooth, consistent movement of the plate as it was kept within the field of the video camera. For delivery of the tap stimuli, a mechanical tapper, which consisted of an electromagnetic relay and a wire arm, was mounted on the holder. The electromagnetic relay was connected to a Grass stimulator (Model S88), which regulated the delivery of each stimulus. The wire arm of the tapper was positioned so that the stimulus was delivered at a location halfway up the side of the dish. The stimulator was set to deliver a $25-\mathrm{msec}, 60-\mathrm{V}$ pulse to the relay.
}

\section{Procedure}

Individual worms were observed on 5-cm Petri plates filled with 10-ml NGM agar and streaked with $E$. coli strain OP50 (Brenner, 1974). In the research on endogenous oscillators, an oscillator has traditionally been split into phase angles, instead of time intervals. Therefore, the defecation intervals were determined for each worm, and the period split into $360^{\circ}$ to determine the time of tap delivery. It was important that the tap stimulus was delivered at a particular place in the cycle. If the tap were administered at a set time interval, rather than at a phase angle, after the last defecation of each animal, it would occur at different phases of the oscillator, since a 4 day-old worm's defecation cycle can range at least from 30 to $60 \mathrm{sec}$ (Croll \& Smith, 1978). For example, administering the tap stimulus at a time equivalent to $330^{\circ}$ into the defecation cycle of one worm could mean that the tap would be given at the beginning of the next cycle for a second worm with a shorter defecation cycle.

Test plates were washed with $E$. coli (streaked) $48 \mathrm{~h}$ before testing: The animals were preplated on the streaked plates $24 \mathrm{~h}$ later and left at room temperature $\left(20^{\circ}-22^{\circ} \mathrm{C}\right)$ overnight. Prior to observation, the animals were placed on the stereomicroscope stage for $10 \mathrm{~min}$, with the light on the stage turned off and the lid of the Petri dish removed. When recording began, the light was turned on; measurements of defecation cycles were recorded from the expulsion step of the defecation cycle until the expulsion of the next defecation cycle.

\section{Scoring}

A worm was considered to have made a reversal response to the tap stimulus if the videotape recording showed that the worm moved backward within 1 sec of the tap stimulus. The magnitude of the reversal was scored by reviewing videotapes, using stopframe video analysis to trace the path of the reversal onto an acetate sheet. If a worm was spontaneously reversing when the tap was administered, that worm was not used in the data for response magnitude across phase groups. The acetate tracings were then digitally scanned and measured (DeskScanII, NIH Image software, Power Macintosh computer). For analysis, the measurements were transferred into a statistical program (StatView 4.0).

\section{EXPERIMENT 1}

The first experiment was designed to investigate the effects of age on the defecation cycle. Thomas (1990) reported an increase in the mean duration of defecation cycles in worms as a function of age: Cycles ranged from $42 \mathrm{sec}$ at age $50 \mathrm{~h}$ to $59 \mathrm{sec}$ at $142 \mathrm{~h}$ of age. Bolanowski, Russell, and Jacobson (1981) observed a rapid decline in defecation frequency from Day 3 to Day 6 of a C. elegans' life. Taken together, these data support the notion that the defecation cycle increases in length as worms age. The studies of habituation in C. elegans have primarily been done using 4-day-old worms (e.g., Rankin et al., 1990; Rankin \& Broster, 1992; Wicks \& Rankin, 1995). The purpose of this experiment was to determine whether the length of the defecation cycle differed significantly between 3 and 5 days of age and, more important for this study, whether it differed significantly over the course of the 4 th day.

\section{Method}

Subjects. A total of 40 hermaphroditic N2 C. elegans were used.

Procedure. The subjects were separated into four groups of 10 worms. The first group consisted of worms that were 3 days old 
tested at $73 \mathrm{~h}$ of age. The second group consisted of 4-day-olds that were tested at $90 \mathrm{~h}$ of age. The third group was made up of 4-dayold worms that were tested at $99 \mathrm{~h}$ of age. The final group consisted of 5-day-old worms that were $126 \mathrm{~h}$ old when they were tested.

The worms were left undisturbed on the microscope stage for $10 \mathrm{~min}$, after which three defecation cycles for each worm were recorded. For each worm, a mean of the three interdefecation intervals (IDIs) was calculated and used as the IDI for that worm.

\section{Results and Discussion}

Figure 1 shows the mean IDI for each of the four groups of subjects. An analysis of variance (ANOVA) showed a significant difference in IDIs across age groups $[F(3,36)=$ $94.078, p<.05]$. Fisher's protected least significant difference tests indicated that the mean IDIs between each group, except that between the $73-\mathrm{h}$ and the 90 -h groups, were significantly different.

These results show that age does in fact affect the duration of the defecation cycle of $C$. elegans, consistent with the research of Bolanowski et al. (1981) and Thomas (1990). In addition, the results indicated that there was a significant difference in the IDIs over the course of Day 4 (groups 90 and $99 \mathrm{~h}$ of age). Since the habituation experiments in our laboratory use 4-day-old worms (e.g., Rankin et al., 1990; Rankin \& Broster, 1992; Wicks \& Rankin, 1995) and since the defecation cycle changes in duration from the beginning to end of the 4th day posthatch, in all further experiments only worms $92-99 \mathrm{~h}$ of age were used.

\section{EXPERIMENT 2}

This experiment was designed to investigate whether a tap affected the defecation cycle by resetting the cycle

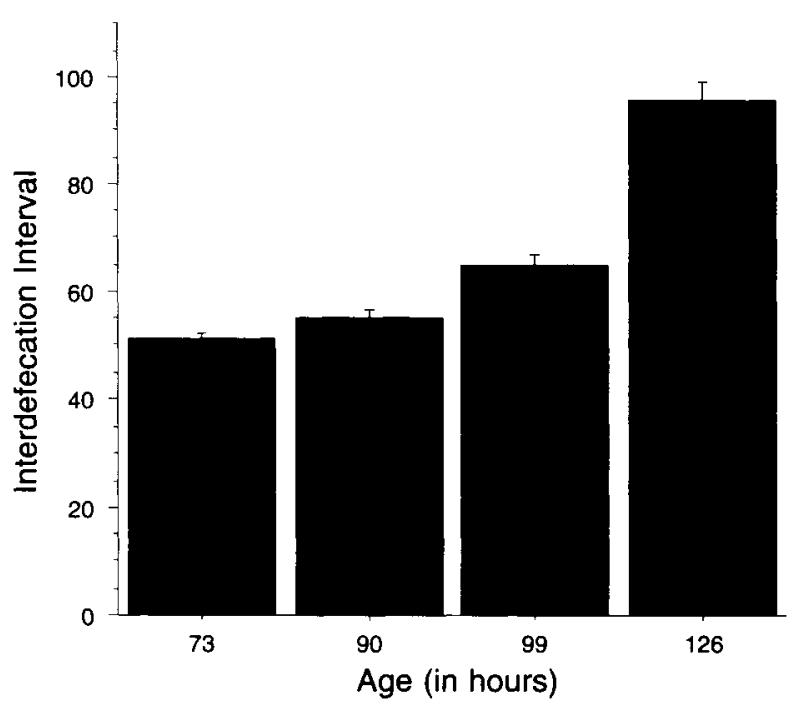

Figure 1. Interdefecation intervals (IDIs, in seconds) for four different age groups ( $N=10$ /group): 3-day-olds ( 73 h old), 4-dayolds made late in the day ( $90 \mathrm{~h}$ old $), 4$-day-olds made early in the day $(99 \mathrm{~h}$ old $)$, and 5 -day-olds (126 h old). With the exception of $73 \mathrm{~h}$ and $90 \mathrm{~h}$, all the groups differed significantly in the duration of the IDIs. in the same way as is done by a touch stimulus (Chalfie et al., 1985; Thomas, 1990) or whether it had any other effects on defecation. In addition, the procedure also tested whether administering a tap stimulus at different points in the defecation cycle of $C$. elegans affected the frequency or magnitude of reversals to tap.

\section{Method}

Subjects. A total of 392 hermaphroditic N2 C. elegans ranging from $92-99 \mathrm{~h}$ of age were used. In addition to age, the defecation cycle can be influenced by a number of environmental factors, such as bacterial contamination, temperature, and humidity. In order to ensure that only healthy subjects were tested, it was important to establish criteria for the length of the IDI used. The lower limit criterion of $41 \mathrm{sec}$ was established using data from Liu and Thomas (1994), who reported that the average defecation cycle was $45.3 \mathrm{sec}$, with a standard deviation of 4.3 seconds. Data from Experiment 1 showed that the average defecation cycle of 4-day-old worms was $60 \mathrm{sec}$, with a standard deviation of $5 \mathrm{sec}$; thus, the upper limit was set at $65 \mathrm{sec}$. Any animals with defecation cycles falling outside of the $41-65$ sec criterion were not used.

Procedure. The experiment consisted of 12 groups of animals, with 31-35 worms in each group. The 12 groups represented the defecation cycle divided into $30^{\circ}$ phases (with 0 indicating the expulsion), in order to determine how stimuli at each phase of the cycle might affect the defecation cycle and how the tap-withdrawal response was affected by taps at each of these phases. Each of the 12 groups corresponded to a different phase in the defecation cycle: $0^{\circ}, 30^{\circ}, 60^{\circ}, 90^{\circ}, 120^{\circ}, 150^{\circ}, 180^{\circ}, 210^{\circ}, 240^{\circ}, 270^{\circ}, 300^{\circ}, 330^{\circ}$.

For each worm tested, the first step was to measure the duration of three defecation intervals. A spreadsheet was used to calculate the average duration of the individual worm's defecation cycle. The average defecation cycle duration was then used to calculate the time that would correspond to each of the phases of the defecation cycle (by dividing the mean duration into $360^{\circ}$ ). This, in turn, was used to determine the time after the third defecation at which the tap stimulus should be administered. The tap stimulus was then delivered at the appropriate time for the appropriate group phase of the individual animal. The final step was to record five defecation cycles following the tap. The five defecation cycles following the tap were then used to calculate the phase change for each worm. The magnitude of reversals was calculated using the measurements taken from the acetate tracings. As a result of technical difficulties in recording and scoring some of the data, the number of worms providing response magnitude data ranged from 25 to 28 per group. ANOVAs were performed on all the data, using an alpha level of $p<.05$.

\section{Results}

The effects of a single tap on the defecation cycle. Tap does have a significant effect on the duration of the defecation cycle, and that effect differs depending on the phase of the cycle in which the tap was delivered. The phase response curve for the first defecation interval after the tap is shown in Figure 2A. If there were no change in duration of the cycle following the tap, the score would be zero. Any score greater than zero represents an increase in the duration of the cycle and, thus, a phase change. Tap stimuli delivered at all phases affected the defecation cycle: Tap had a small effect on the phase of the defecation cycle immediately following the defecation, and the effect on the phase gradually increased until $240^{\circ}$ into the defecation cycle, at which time there was a sharp drop in the effect on phase change just prior to the next defecation. An ANOVA comparing the phase 
A
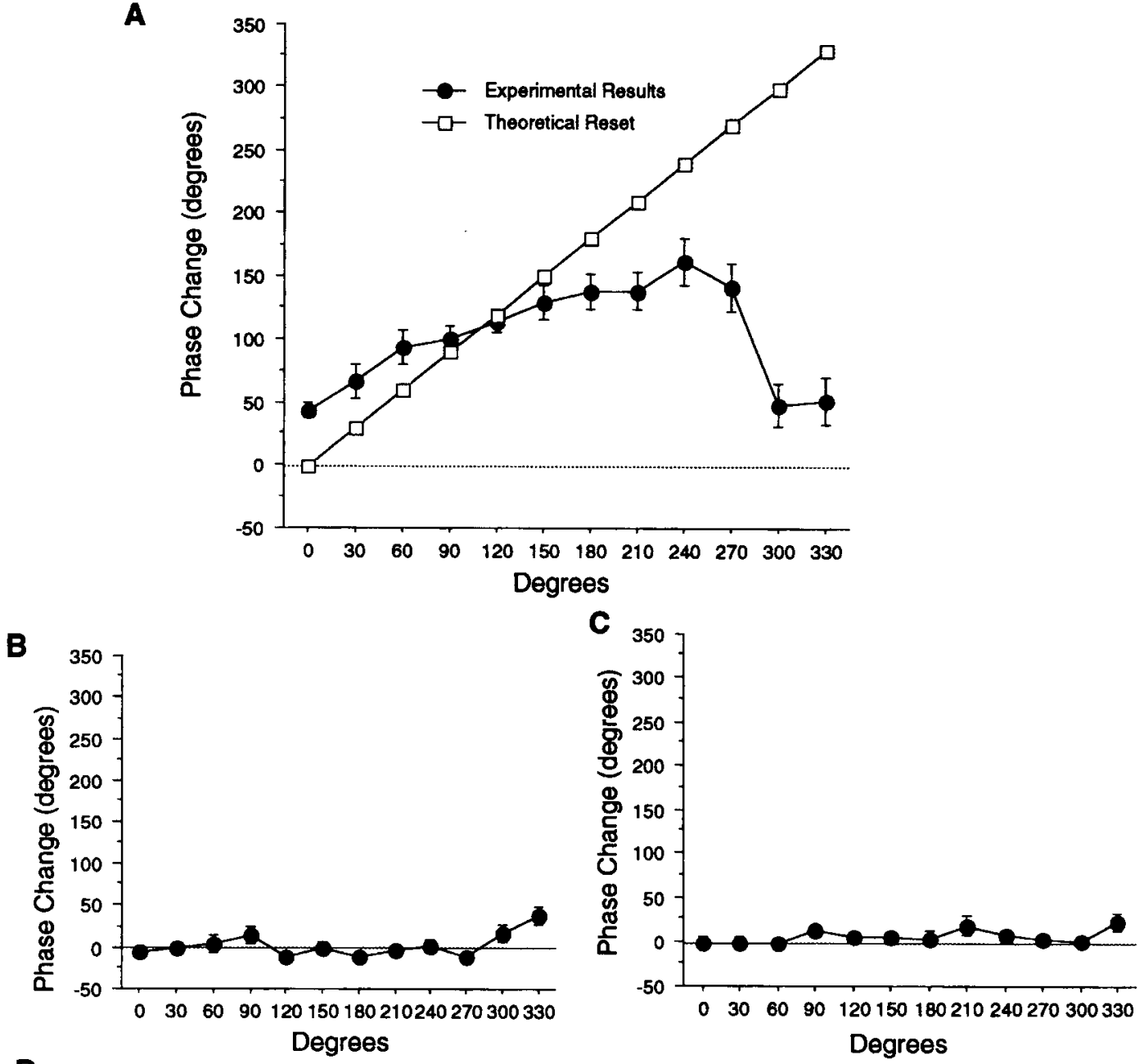

D

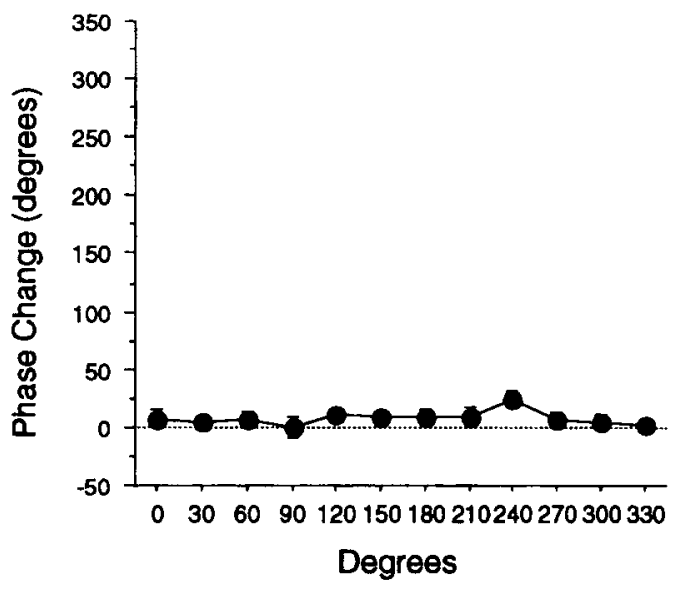

$\mathbf{E}$

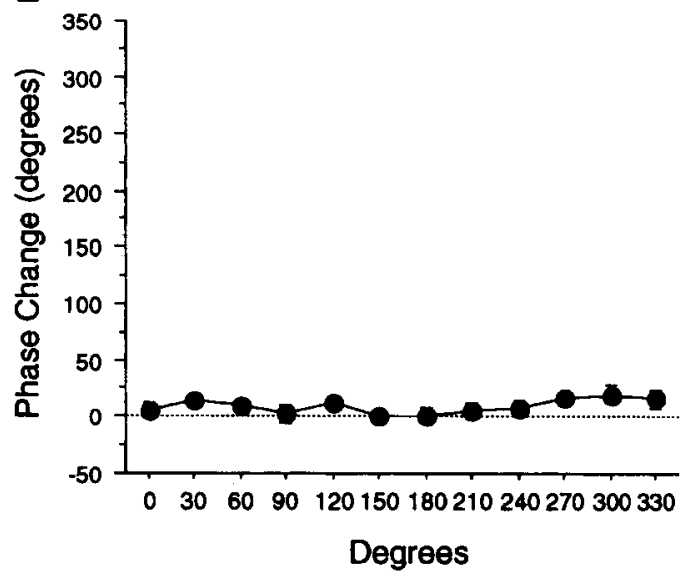

Figure 2. Mean phase changes in response to tap delivered at each $30^{\circ}$ phase of the defecation cycle $(N=31-35$ per group) for the first through the fifth defecations after a tap. In the first cycle after tap, there were significant differences in the phase changes, depending on where the tap was delivered during the defecation cycle. There were no significant differences in the effects of the tap at different phases of the defecation cycle for the second through the fifth cycles after the tap. (A) Mean phase change of the first defecation cycle after the tap (filled circles). Open boxes represent the theoretical results predicted if the tap caused reset of the defecation cycle at all phases. (B) Mean phase change of the second defecation cycle after the tap. (C) Mean phase change of the third defecation cycle after the tap. (D) Mean phase change of the fourth defecation cycle after the tap. (E) Mean phase change of the fifth defecation cycle after the tap. 
change across groups showed that there were significant differences in the degree of phase change, depending on when in the cycle the tap was delivered $[F(11,368)=$ $8.381, p<.05]$.

If the defecation cycle was entrained to some "master oscillator," one might predict that after being disrupted, the cycle would gradually shift back into phase over the next few cycles. The phase response curves for the second through the fifth defecation intervals following the tap are shown in Figures 2B, 2C, 2D, and 2E. Although the tap seems to have an effect on the length of the defecation cycle immediately following the tap stimulus (Figure $2 \mathrm{~A}$ ), the tap does not appear to have an effect on the length of the second, third, fourth, or fifth defecation cycle following the tap. These results suggest that the tap causes the endogenous oscillator regulating defecation to permanently shift out of phase.

Thomas (1990) reported that the touch stimulus caused a reset in the defecation cycle (i.e., caused the cycle to begin again). In Figure 2A, the idealized data for reset of the phase of the defecation cycle are plotted along with the data from this experiment, showing the response of the defecation cycle to tap stimuli. The data show that the tap only resets the defecation cycle when administered at $90^{\circ}, 120^{\circ}$, and possibly $150^{\circ}$. In order for the tap to reset the defecation cycle in each phase group, the points of the two lines in Figure 2A would need to be coincident with each other.

The effects of phase of the defecation cycle on response to a single tap. An examination of the withdrawal responses to taps delivered at different phases of the defecation cycle showed that the phase of the defecation cycle at which the tap was delivered did not have a significant effect on the magnitude (Figure 3A) or the frequency (Figure 3B) of reversals. Although the responses appear to be smaller around the time of the defecation $\left(0^{\circ}, 30^{\circ}\right.$, and $\left.330^{\circ}\right)$, an ANOVA on the magnitude of the reversals showed no significant differences between groups $[F(11,290)=0.810, p>.05]$. Thus, variability in either the frequency or the magnitude of the response to a tap is not greatly influenced by the placement of the tap with regard to phases of the defecation cycle.

\section{EXPERIMENT 3}

This experiment was designed to investigate whether multiple taps affect the defecation cycle by causing a constant phase change in the defecation cycles following each tap or whether they have any other effects on the defecation cycle. In addition, the data test whether administering multiple tap stimuli at a particular phase in the defecation cycle of $C$. elegans affects the magnitude of the tap-withdrawal response and, consequently, affects the shape of the habituation curve by decreasing some of the variability often observed when a tap is administered at a given ISI, irrespective of the defecation cycle.

\section{Method}

Subjects. A total of 66 hermaphroditic N2 C. elegans was used. The same criteria for the length of the IDI as those described in Experiment 2 were also used in this experiment.

Procedure. For all the subjects, three defecation cycles were initially recorded in order to determine the mean defecation cycle for each individual subject. The mean defecation cycle was then used to determine when, following each defecation, the tap should be administered for the experimental subjects, using a spreadsheet: The worms were divided into two groups, one for each phase tested. The phases of the defecation cycle chosen for tap delivery were $120^{\circ}$ and $270^{\circ}$. These phases were chosen to represent phases one third and two thirds of the way through the defecation cycle. Experiment 2 showed that a tap delivered at $120^{\circ}$ caused a reset of the cycle, whereas a tap delivered at $270^{\circ}$ caused a large phase change but did not reset the cycle. Sixteen experimental worms from the first group $\left(120^{\circ}\right.$ group) received the tap stimuli $120^{\circ}$ into the defecation cycle, and 17 experimental worms from the second group $\left(270^{\circ}\right.$ group) received the tap stimuli $270^{\circ}$ into the defecation cycle. Each of the experimental worms in each group $\left(120^{\circ}\right.$ and $\left.270^{\circ}\right)$ was paired with a yoked control worm that was given taps at the same intertap intervals as its experimental counterpart, regardless of the length or phase of its own defecation cycle.

Each subject received 30 stimuli. The lengths of two defecation intervals following the end of the 30 taps were also recorded.

During Experiment 2, there were no defecation intervals greater than $133 \mathrm{sec}$ recorded. Therefore, for this experiment, if more than $133 \mathrm{sec}$ went by without a defecation, it was assumed that either the experimenter had missed one or the worm had moved up the side of the Petri plate out of the food, interrupting the rhythmic defecation. For the purpose of data analysis, these intervals were considered missing data points (less than $4 \%$ of the total data points). There were 16 experimental worms for the $120^{\circ}$ group and 17 experimental worms for the $270^{\circ}$ group.

\section{Results}

Effects of multiple taps on the defecation cycle. For both groups tested, the first tap in the series caused an increase in the length of the defecation cycle over the baseline defecation cycle similar to that which was reported in Experiment 2. With repeated stimulation, the phase shift of the IDI habituated for both the $120^{\circ}$ and the $270^{\circ}$ groups (Figure 4A). To test for habituation, the mean of the first two IDIs was compared with the mean of the last two IDIs, using a paired $t$ test (Figure 4B). Both ISI groups showed significant decrement from first to last IDIs [120 group: $t(15)=3.12, p=.007 ; 270^{\circ}$ group: $t(16)=2.08$, $p=.05]$. Although both groups showed significant decrement in the amount of phase change from the first tap to the last tap, this decrement was modest, with only a $16.53 \%$ drop in the $120^{\circ}$ group and only a $14.13 \%$ drop in the $270^{\circ}$ group. Immediately after the end of stimulation, the IDI in both groups returned to its prehabituation duration (Figure 4A).

Effects of phase of the defecation cycle on response to multiple taps. The habituation curves for response magnitude to tap for the four groups of worms are shown in Figures 5A-5D. There were 16 worms in each group (data from one of the $270^{\circ}$ control worms were lost, owing to computer error; therefore, its experimental partner's data were discarded). In order to compare the variability 
A

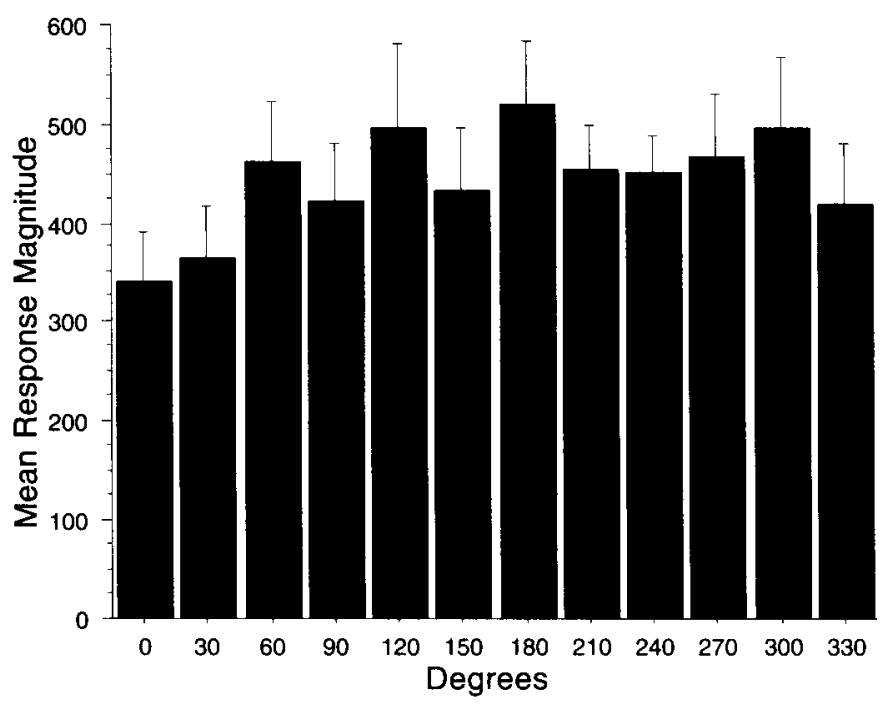

B

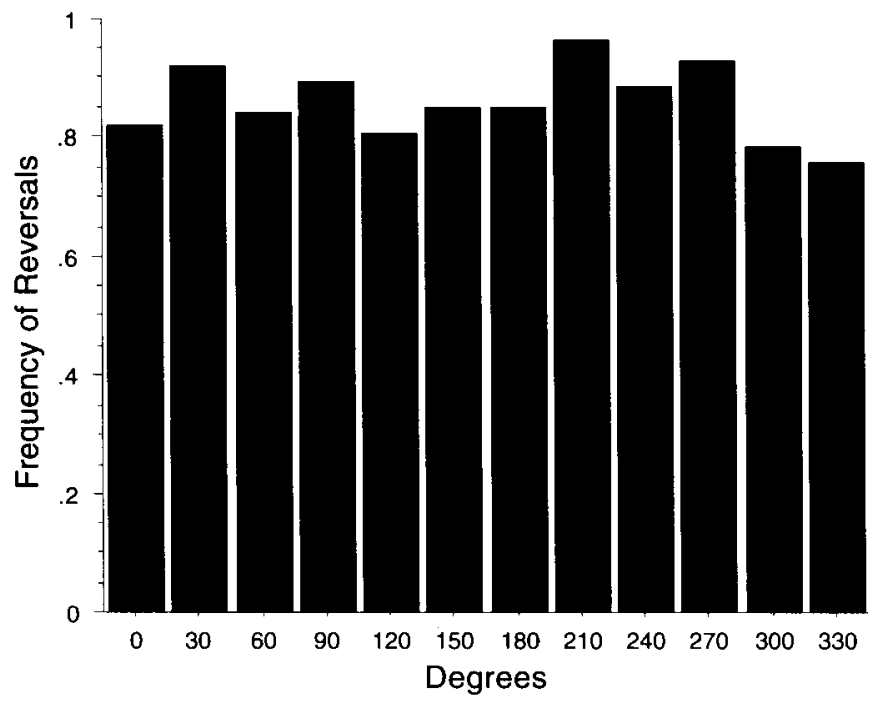

Figure 3. (A) Mean response magnitude of the tap-withdrawal response for each group. There were no significant differences in tap response magnitude, regardless of the phase of the defecation cycle when a tap was delivered. (B) The frequency of reversals to tap for taps delivered at the different phases of the defecation cycle.

between the habituation groups, an ANOVA was run on the mean absolute deviation scores for each group. The mean absolute deviation scores were calculated by determining the absolute value of the difference between the mean scores for each stimulus and each animal's score on that stimulus. This comparison of deviations between responses to taps delivered at a set phase of an individual worm's defecation cycle with responses to taps delivered without reference to an individual's cycle shows that administering taps at a set phase of the defecation cycle did not affect the variability of the tap-withdrawal response of $C$. elegans [Figure $5 \mathrm{E} ; F(3,116)=0.614$, $p=.607]$.

\section{GENERAL DISCUSSION}

\section{The Effects of Tap on the Defecation Cycle}

Tap does cause a phase change in the defecation cycle. Studies of other endogenous oscillators have shown that a disruptive stimulus can cause either a phase advance, 


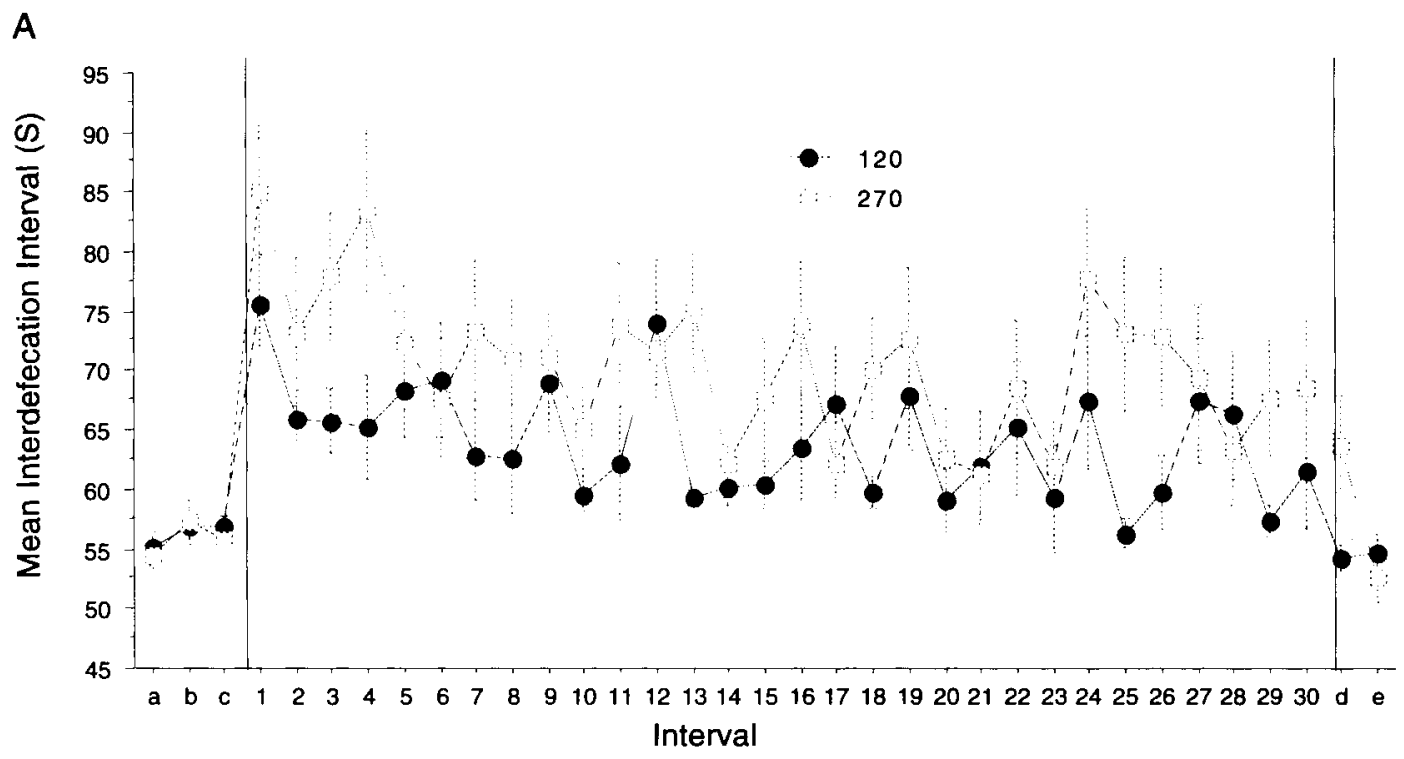

B

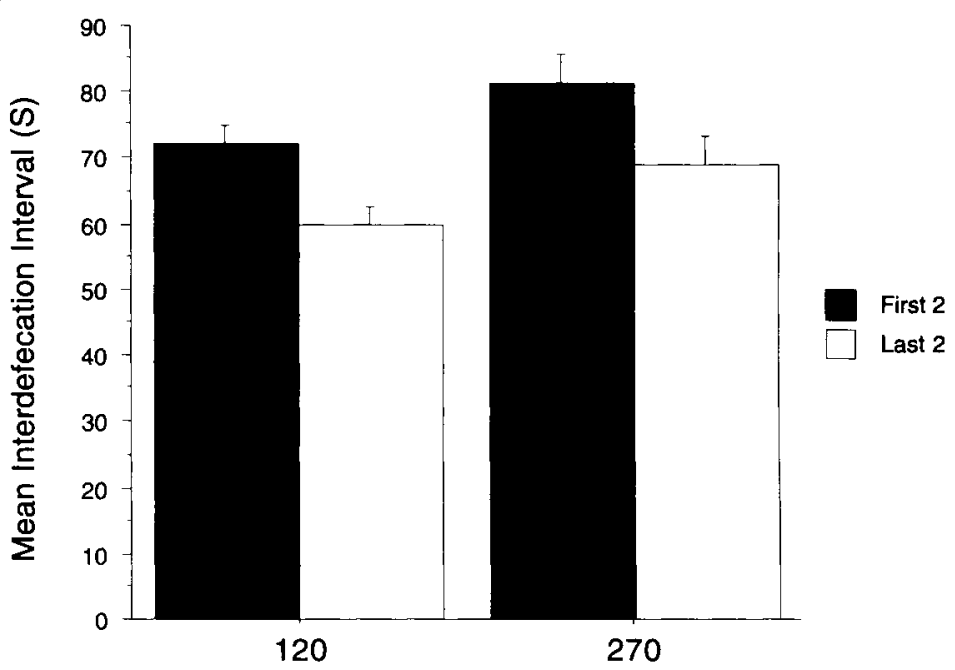

Figure 4. (A) The mean interdefecation intervals (IDIs) for the $120^{\circ}$ (filled circles; $N=16$ ) and $270^{\circ}$ (open boxes; $N=17$ ) experimental groups. The first three points for each group represent 3 baseline IDIs prior to stimulation; they are followed by 30 IDIs during habituation training to 30 taps. The last two points on the curves represent the first 2 IDIs following the end of the tap stimulation. (B) The mean of the first 2 IDIs and the mean of the last 2 IDIs for the $120^{\circ}$ and the $270^{\circ}$ groups. Within each group, there was a significant decrement in the duration of the IDI from the beginning of tap habituation training to the end.

where the cycle would become shorter than the mean, or a phase delay, where the cycle would become longer than the mean. The effects of the disruptive stimulus depend on the intensity of the stimulus and the initial phase of application (Broda, Johnson, Taylor, \& Hastings, 1989). In this study, the smallest phase change occurs close to the time of defecation, between $10 \mathrm{sec}$ before and $10 \mathrm{sec}$ after the expulsion phase of the defecation. The greatest phase change occurs in the second half (Figure 2A) of the defecation cycle, at $240^{\circ}$. The data, therefore, show that the tap stimulus has a different effect on the defecation cycle when it occurs at different times during the cycle.

Thomas (1990) reported that administering a touch stimulus to the head of the worm produced a reset in the endogenous oscillator. If a touch was administered $20 \mathrm{sec}$ into the defecation cycle, the following defecation cycle was $20 \mathrm{sec}$ longer, suggesting a reset to time zero at the time of the touch stimulus. If that were the case for tap, there would have been an increase in phase change corresponding to the higher phase change groups. However, 
A
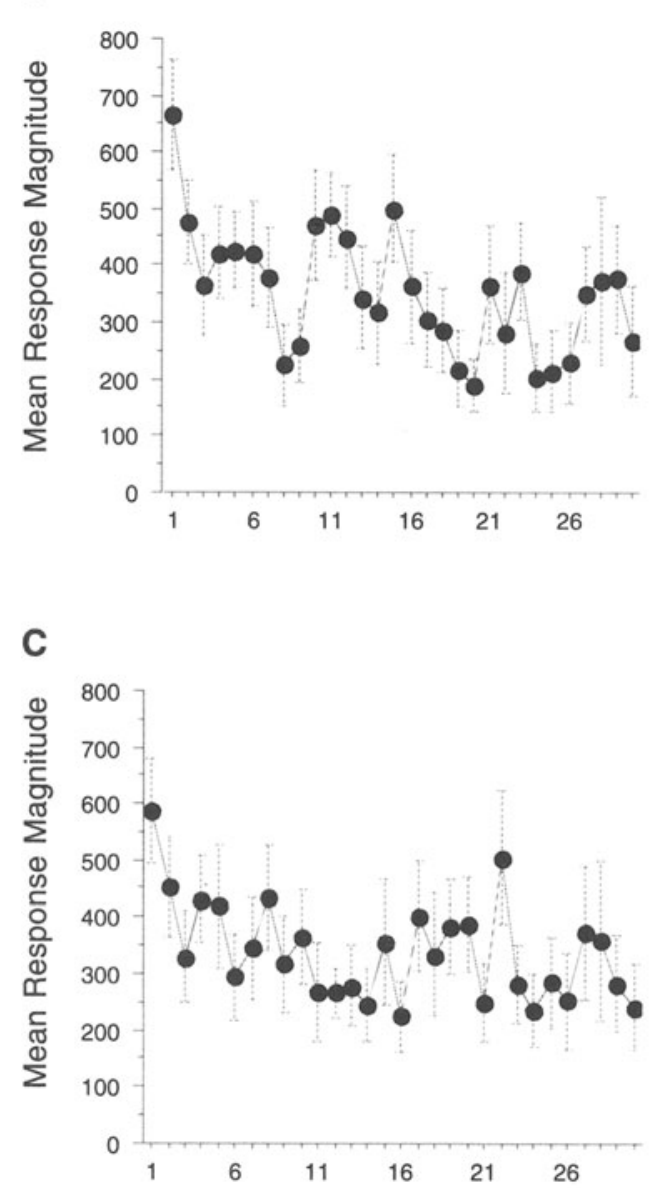

B

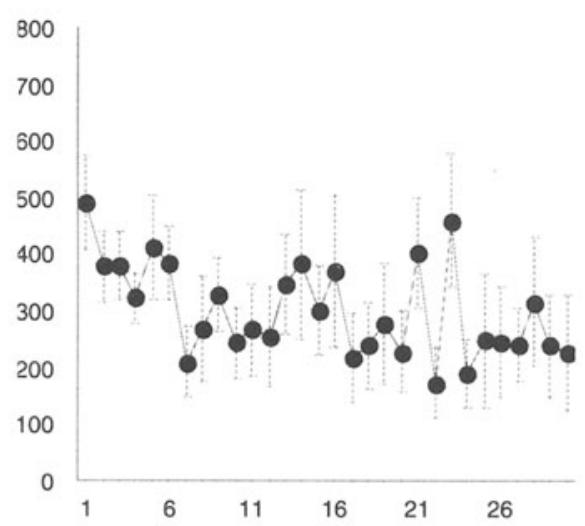

D

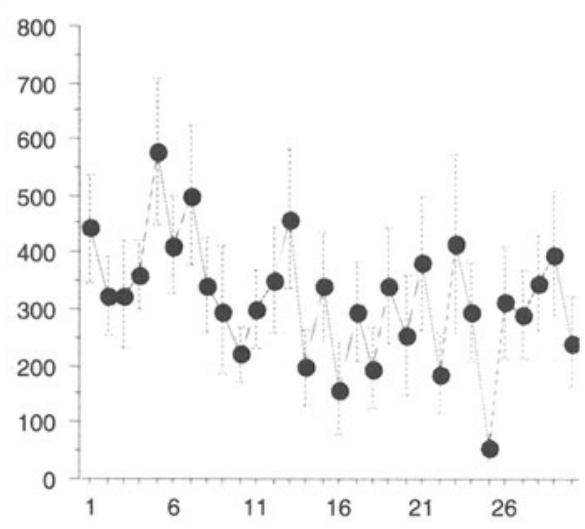

E

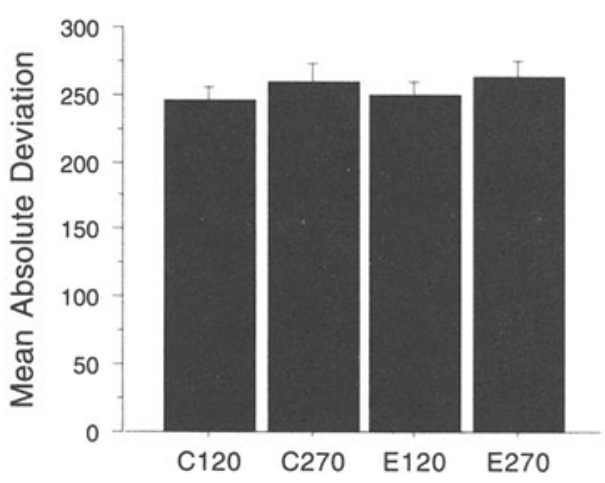

Figure 5. (A) Habituation of the tap-withdrawal response of the $120^{\circ}$ experimental group $(N=16)$ when the tap was delivered at $120^{\circ}$ into the defecation cycle. (B) Habituation of the tap-withdrawal response of the $120^{\circ}$ yoked control group $(N=16)$. (C) Habituation of the tap-withdrawal response of the $270^{\circ}$ experimental group $(N=16)$. (D) Habituation of the tap-withdrawal response of the $270^{\circ}$ yoked control group $(N=16)$. (E) Mean absolute deviation scores (absolute value of mean scores - individual scores) for each of the four groups.

with tap there was an increase in phase change up to $240^{\circ}$ in the defecation cycle, at which time there was a sharp decrease in the phase change. The increasing phase change from $30^{\circ}$ to $240^{\circ}$ was not proportional to the phase angle at which the tap was administered. If the tap stimulus produced a reset in the oscillator, there should have been phase changes equal to the phase group we were testing. For example, if the tap was given $5 \mathrm{sec}$ after expulsion, the next defecation cycle should have been $5 \mathrm{sec}$ longer. However, the only groups to display a phase change equal 
to its phase were the $90^{\circ}, 120^{\circ}$, and $150^{\circ}$ groups, and therefore these were the only groups considered to display a full resetting of the defecation cycle (Figure $2 \mathrm{~A}$ ).

There are several differences between the method used by Thomas (1990) and the present method that might account for the different results. These include the type of stimulus used, the timing of the stimulation, and the number of stimuli tested. Thomas (1990) used a headtouch stimulus, whereas the present study used a tap stimulus. The head-touch stimulus only activates the head-touch circuit (Chalfie et al., 1985). The tap stimulus involves a broad neural circuit activating both the head- and the tail-touch circuits simultaneously (Wicks $\&$ Rankin, 1995). Therefore, it is possible that the two different stimuli have different effects on the defecation cycle. In addition, the touch stimulus used by Thomas was delivered by hand and, therefore, may have varied in intensity with each delivery. Variations in the intensity of the stimulus may have resulted in different effects on the defecation cycle. Thomas administered the touch stimulus at specific time intervals in the defecation cycle, rather than at different phases, at only four times in the defecation cycle $(20,25,30$, and $35 \mathrm{sec}$, reflecting phase angles of approximately $150^{\circ}, 210^{\circ}, 240^{\circ}$, and $270^{\circ}$, respectively, if the cycle was $45 \mathrm{sec}$ long), all of which were quite close to each other, and only during the second half of the defecation cycle, rather than spanning the entire defecation cycle.

The data from the present study indicate that tap caused the oscillator to shift out of phase (Figure 2A) and stay out of phase (Figure 2B, 2C, 2D, and 2E), causing a longlasting phase delay. The first defecation cycle after the tap was significantly longer than the mean defecation cycle preceding the tap (Figure 2A). However, the second through the fifth defecations after the tap were all approximately the same length as the mean defecation cycles before the tap (Figures 2B, 2C, 2D, and 2E). If the defecation cycle were to shift back into phase, at least one of the $2 \mathrm{nd}, 3 \mathrm{rd}, 4 \mathrm{th}$, or 5 th defecation cycles would be shorter or longer than the mean defecation cycle preceding the tap, in order to shift back into phase. However, since this did not occur in the data, it is clear that the tap stimulus caused a long-lasting phase delay in the defecation cycle of $C$. elegans and that, within five cycles, there was no recovery to the original phase. The return to the original length of the defecation cycle extends Thomas' (1990) finding that the 2 nd defecation after the touch stimulus was the same length as the mean defecation cycle prior to the touch stimulus. From these data, it is possible to conclude that the defecation cycle is not strongly entrained to some internal oscillator with which it would have to retain a consistent phase relationship.

Studies of circadian biological oscillators have shown that (1) most circadian clocks shift phase in response to light pulses and, therefore, rarely produce a complete reset and (2) the magnitude of the shift is dependent on when the stimulus is delivered (Aschoff, 1965). Therefore, our results, which did not show resetting of the defecation cycle caused by the tap stimulus, correspond quite closely to Aschoff's description of circadian clocks: Our results showed that the endogenous oscillator shifted phase and that the magnitude of the shift was dependent on when the stimulus was delivered and rarely produced a complete reset.

The effects of multiple taps on the defecation cycle provide evidence that the phase change caused by a disruptive stimulus habituated with repeated stimulations. The IDI gradually decreased toward the baseline IDI with repeated presentations of the tap stimulus. Interestingly, 25 out of the 30 IDIs following tap were longer in the $270^{\circ}$ group than in the $120^{\circ}$ group. This observation is consistent with the results of Experiment 2, where we found a phase change that was approximately $25^{\circ}$ greater in the $270^{\circ}$ group than in the $120^{\circ}$ group. Experiment 3 provided evidence that this increased phase change continued across multiple taps and that even though the phase change habituated for both groups, the phase change still remained greater for the $270^{\circ}$ group throughout the experiment.

The results of Experiment 3 show that the disruption of the defecation cycle followed the same mechanisms of habituation as other responses, such as the tap-withdrawal response and the touch response, as follows: (1) Similar to the decreased tap-withdrawal and touch responses with multiple stimulus presentations, the phase change of the defecation cycle was elicited less with each tap given, and (2) similar to the habituation curves of the tapwithdrawal response and the touch response, the habituation curve of the IDI also showed some variability (Figure 4A).

Effects of phase of the defecation cycle on response to tap. The phase of the defecation cycle in which a tap was delivered had no significant effect on the tapwithdrawal response when only a single tap was administered. There was a small trend apparent in the data (Figure $3 \mathrm{~A}$ ), however, that indicated that response magnitude slowly increased from the time of the expulsion until the middle of the defecation cycle at $180^{\circ}$, at which time the response magnitude decreased again. Figure 3B shows a small decrease in the frequency of reversals right before the next defecation would be about to occur. A decrease in responding near the end of the defecation cycle would be expected, because tail muscles needed for reversing are now involved in defecation (Thomas, 1990).

If the interaction between time of tap delivery and the phase of the defecation cycle played an important role in response magnitude during habituation, we would expect less variation in the habituation curve in the experimental group (Figures $5 \mathrm{~A}-5 \mathrm{C}$ ), where taps were administered according to each individual worm's defecation cycle, than in the control groups (Figures 5B and 5D), where taps were given regardless of each animal's defecation cycle.

It is possible that the attempt to design an experiment in which the tap was delivered at a consistent phase of the defecation cycle was not successful. Once the first tap was administered, the defecation cycle was shifted out of phase and, therefore, was longer than the baseline defecation cycle. This was not taken into account when 
calculating the time for administering the second, third, fourth, and successive taps. For example, if the mean IDI prior to the tap stimulus was $60 \mathrm{sec}$, tap was administered $20 \mathrm{sec}$ after every defecation for the $120^{\circ}$ group. This did not take into account the length of the new defecation cycle. If the reset IDI was closer to $80 \mathrm{sec}$ and the tap was still administered $20 \mathrm{sec}$ after the expulsion, the tap would then correspond more closely to a phase of $90^{\circ}$. Since the phase length varied over the course of the habituation series, the tap ranged over a portion of the defecation cycle, rather than being administered at a distinct phase. Our original hypothesis about the interaction between an internal oscillator and response magnitude to tap was predicated on a stable period oscillator. In this experiment, we have demonstrated that the period of the defecation cycle is altered by a single tap and is altered in a variable fashion by repeated taps. Thus, the period becomes a "moving target" as it first resets and the reset then habituates slowly to repeated taps. This may contribute to even greater variability of responding than with a stable period oscillator. It is possible that if the timing of each tap had been determined more precisely, we would have obtained habituation curves with less variability than those obtained in Experiment 3 . It may be possible in future experiments to take this into account.

\section{Conclusions}

Our findings suggest that tap stimuli have a large effect on the defecation cycle. When a single tap was administered, there was a phase change that caused a longlasting phase delay in the defecation cycle. The length of the phase delay depended on where in the defecation cycle the tap was administered. The defecation cycle was only reset when the tap was delivered at $120^{\circ} \pm 30^{\circ}$. When multiple taps were administered, the phase change caused by the tap habituated slowly across the course of the experiment.

With respect to the effect of the phase of the defecation cycle on the tap-withdrawal response, there was a weak relationship. The results of Experiment 2 indicate that the phase of the defecation cycle at which a tap is administered has no consistent effect on the magnitude or frequency of the tap-withdrawal response. Repeated stimulation at a consistent phase of the defecation cycle did not appear to have any effect on the variability observed in the habituation curves. It may be that the oscillator underlying defecation does not play a role in the response to tap but that some other, as yet unidentified, oscillator does influence response to tap, or it may be that the source of variability in the response to tap is independent of internal oscillators. Further investigation with a variety of organisms is needed.

\section{REFERENCES}

Aschoff, J. (1965). Response curves in circadian periodicity. In J. Aschoff(Eds.), Circadian clocks (pp. 95-111). Amsterdam: NorthHolland.

BolanoWSKi, M. A., Russell, R. L., \& JACOBSON, L. A. ( 1981). Quantitative measures of aging in the nematode $C$. elegans: I. Population and longitudinal studies of two behavioral parameters. Mechanisms of Aging \& Development, 15, 279-295.

BRENNER, S. (1974). The genetics of Caenorhabditis elegans. Genetics, 77, 71-94.

Broda, H., Johnson, C. H., Taylor, W. R., \& Hastings, J. W. (1989). Temperature dependence of phase response curves for drug-induced phase shifts. Journal of Biological Rhythm, 4, 327-333.

Chalfie, M., Sulston, J. E., White, J. G., Southgate, E., Thomson, J. N., \& BRENNER, S. (1985). The neural circuit for touch sensitivity in Caenorhabditis elegans. Journal of Neuroscience, 5, 956-964

Chiba, C. M., \& Rankin, C. H. (1990). A developmental analysis of spontaneous and reflexive reversals in the nematode Caenorhabditis elegans. Journal of Neurobiology, 21, 543-554.

Croll, N. A., \& Smith, J. M. (1978). Integrated behaviour in the feeding phase of Caenorhabditis elegans (Nematoda). Journal of Zoology, 184, 507-517.

Davis, M., Parisi, T., Gendelman, D. S., Tischler, M. D., \& Kehne, J. H. (1982). Habituation and sensitization of "startle" responses elicited electrically from the brainstem. Science, 218, 688-690.

Galloway, J. A., Wicks, S. R., \& Rankin, C. H. (1997). Neural integration of external stimuli with internal processes. Society for Neuroscience Abstracts, 23, 1337.

Groves, P. M., \& Thompson, R. F. (1970). Habituation: A dual-process theory. Psychological Review, 77, 419-450.

Iwasaki, K., \& Thomas, J. H. (1997). Genetics in rhythm. Trends in Genetics, 13, 111-115.

LıU, D. W. C., \& Thomas, J. H. (1994). Regulation of a periodic motor program in C. elegans. Journal of Neuroscience, 14, 1953-1962.

Peeke, H. V. S., \& Veno, A. (1973). Stimulus specificity of habituated aggression in three-spined sticklebacks (Gasterosteus aculeatus). Behavioral Biology, 8, 427-431.

Rankin, C. H., BeCK, C. D. O., \& Chiba, C. M. (1990). Caenorhahditis elegans: A new model system for the study of learning and memory. Behavioural Brain Research, 37, 89-92.

RANKIN, C. H., \& BRoster, B. S. (1992). Factors affecting habituation and recovery from habituation in the nematode Caenorhabditis elegans. Behavioral Neuroscience, 106, 239-249.

RANKIN, C. H., \& CAREW, T. J. (1987). Development of learning and memory in Aplysia: II. Habituation and dishabituation. Journal of Neuroscience, 7, 133-143.

Reiner, D. J., \& ThOMAS, J. H. (1995). Reversal of a muscle response to GABA during $C$. elegans male development. Journal of Neuroscience, 15, 6094-6102.

Reiner, D. J., Weinshenker, D., \& Thomas, J. H. (1995). Analysis of dominant mutations affecting muscle excitation in Caenorhabditis elegans. Genetics, 141, 961-976.

Thomas, J. H. (1990). Genetic analysis of defecation in Caenorhabditis elegans. Genetics, 124, 855-872.

Thomas, J. H. (1994). The mind of a worm. Science, 264, 1698-1699.

WICKS, S. R., \& RANKIN, C. H. (1995). Integration of mechanosensory stimuli in Caenorhabditis elegans. Journal of Neuroscience. 15. 2434-2444.

(Manuscript received October 25, 1999; revision accepted for publication July 10,2000 .) 